\title{
WEYL GROUPS AND ABELIAN VARIETIES
}

\author{
ANGEL CAROCCA, VÍCTOR GONZÁLEZ-AGUILERA, AND \\ RUBÍ E. RODRÍGUEZ
}

\begin{abstract}
Let $G$ be a finite group. For each integral representation $\rho$ of $G$ we consider $\rho$-decomposable principally polarized abelian varieties; that is, principally polarized abelian varieties $(X, H)$ with $\rho(G)$-action, of dimension equal to the degree of $\rho$, which admit a decomposition of the lattice for $X$ into two $G$-invariant sublattices isotropic with respect to $\Im H$, with one of the sublattices $\mathbb{Z} G$-isomorphic to $\rho$.

We give a construction for $\rho$-decomposable principally polarized abelian varieties, and show that each of them is isomorphic to a product of elliptic curves.

Conversely, if $\rho$ is absolutely irreducible, we show that each $\rho$-decomposable p.p.a.v. is (isomorphic to) one of those constructed above, thereby characterizing them.

In the case of irreducible, reduced root systems, we consider the natural representation of its associated Weyl group, apply the preceding general construction, and characterize completely the associated families of principally polarized abelian varieties, which correspond to modular curves.
\end{abstract}

\section{InTRODUCTION}

It is known that every finite group acts on some curve, hence on some Jacobian, and therefore on some principally polarized abelian variety (p.p.a.v.). However, there is no explicit way of relating the order of the group and the dimension of the p.p.a.v. on which it acts.

For any integral representation of a finite group $G$ there exists a family of principally polarized abelian varieties of dimension equal to the degree of the representation, each invariant under the given action of $G$.

In particular, every root system of rank $n$ has a natural lattice in $\mathbb{R}^{n}$ associated to it, and hence a natural integral representation of its associated Weyl group of degree $n$. Therefore, we may apply the preceding construction to build a family of p.p.a.v.'s of dimension $n$ admitting the given Weyl group (more generally, the full automorphism group of the root system) as subgroup of its group of automorphisms.

2000 Mathematics Subject Classification. Primary 14K99, 14J15; Secondary 32G13, 20C10.

Key words and phrases. Abelian varieties, Weyl groups, integral representations of finite groups, modular curves.

This research was partially supported by the Presidential Science Chair on Geometry and by FONDECYT Grants \# 1030595, 1040319 and 1011039. 
In the case of irreducible, reduced root systems, we show that each p.p.a.v. in the corresponding family is isomorphic (as a complex torus) to a product of elliptic curves. These results are related to an invariant theory developed in the work of E. Looijenga [8] where he also deals with abelian varieties associated to each irreducible root system, all of which admit the action of the corresponding Weyl group and are isomorphic (as complex tori) to a product of elliptic curves.

In general, the reducibility or irreducibility of a given p.p.a.v. (as p.p.a.v.) is not an easy problem. Here we show that, in most cases, our varieties are irreducible as p.p.a.v.'s.

In addition, in the case of irreducible, reduced root systems, we characterize the corresponding families in the moduli space of p.p.a.v.'s $\mathcal{A}_{n}$, and show that they are uniformized by explicit modular curves.

In Section 1 we recall some basic results on principally polarized abelian varieties and roots systems in order to fix the notation.

In Section 2 we consider any integral representation $\rho$ of a finite group $G$, and construct p.p.a.v.'s $(X, H)$, of dimension equal to the degree of $\rho$, which admit a decomposition of the lattice for $X$ into two $G$-invariant sublattices isotropic with respect to $\Im H$, with one of the sublattices $\mathbb{Z} G$-isomorphic to $\rho$. We call these p.p.a.v.'s $\rho$-decomposable. We also show that each $\rho$-decomposable p.p.a.v. is isomorphic to a product of elliptic curves.

Conversely, if $\rho$ is absolutely irreducible, we show that each $\rho$-decomposable p.p.a.v. is (isomorphic to) one of those constructed above, thereby characterizing them.

In Section 3, we apply our characterization to each irreducible, reduced root system and obtain the corresponding Riemann matrices. We also discuss the relationship between our varieties and the previous work of Looijenga, and the notion of dual polarization developed by Birkenhake and Lange [1].

Section 4 describes explicit isomorphisms of our principally polarized abelian varieties with products of elliptic curves and discusses their irreducibility as principally polarized abelian varieties.

Section [5] shows that our families for irreducible, reduced root systems are uniformized by modular curves, which are described explicitly, and complete their connection with the previous work of Looijenga.

Now we fix the notation as follows. A complex torus of dimension $n$ will be by definition a quotient $X \simeq \mathbb{C}^{n} / L$ of the vector space $\mathbb{C}^{n}$ modulo a lattice $L$ of maximal rank. $X$ is called an abelian variety if it admits a polarization; that is, a positive definite hermitian form $H$ on $\mathbb{C}^{n}$ whose imaginary part is integral valued on $L$. In this case, the lattice $L$ always admits a basis such that the matrix of $\Im H$ in this basis is of the form $\left(\begin{array}{rr}0_{n} & D \\ -D & 0_{n}\end{array}\right)$ with a diagonal matrix $D=\operatorname{diag}\left(d_{1}, d_{2}, \ldots, d_{n}\right)$ and positive integers $d_{i}$ with $d_{i} / d_{i+1}$. The $n$-tuple $\left(d_{1}, d_{2}, \ldots, d_{n}\right)$ is called the type of the 
polarization, the integer $d=d_{1} d_{2} \ldots d_{n}$ is called the degree of the polarization and a polarization of type $(1,1, \ldots, 1)$ is called a principal polarization.

The automorphisms of the complex torus $X$ that respect the polarization $H$ form a finite group denoted by $\operatorname{Aut}_{H}(X)$. If $G$ is a subgroup of $\operatorname{Aut}_{H}(X)$, we will say that $(X, H)$ has a $G$-action. The representation of $\operatorname{Aut}_{H}(X)$ on the lattice of $X$ will be called the rational representation of $\operatorname{Aut}_{H}(X)$.

A principally polarized abelian variety of dimension $n$ (p.p.a.v.) will be a pair $(X, H)$ where $X$ is an $n$-dimensional complex torus and $H$ is a principal polarization on $X$. Alternatively we can consider a principally polarized abelian variety $(X, H)$ as a pair $(X, \mathcal{L})$ where $\mathcal{L}$ is a line bundle on $X$ such that its first Chern class $c_{1}(\mathcal{L}) \simeq H$, but in order to give explicit descriptions we will follow the classical terminology.

Each Riemann matrix $Z$ in the Siegel space $\mathbb{H}_{n}$ of degree $n$ gives a p.p.a.v. of dimension $n$. If we denote by $\operatorname{Sp}(2 n, \mathbb{Z})$ the symplectic group, then the complex analytic space $\mathcal{A}_{n}=\mathbb{H}_{n} / \operatorname{Sp}(2 n, \mathbb{Z})$ parametrizes the (isomorphism classes of) p.p.a.v.'s of complex dimension $n$.

Since there are different kinds of equivalences between Riemann matrices or abelian varieties, and so as to avoid ambiguities, we recall the definitions involved in our results.

Two p.p.a.v.'s $\left(X_{1}, H_{1}\right)$ and $\left(X_{2}, H_{2}\right)$ are called isogenous if there exist a surjective homomorphism $h: X_{1} \rightarrow X_{2}$ (as complex tori) with 0-dimensional kernel; they are called isomorphic if the kernel of $h$ is trivial. They will be called isomorphic as principally polarized abelian varieties if there exists an isomorphism $h$ (as above) which further preserves the given polarizations $H_{1}$ and $H_{2}$.

A p.p.a.v. is called irreducible if it is not isomorphic as a principally polarized abelian variety to the product of p.p.a.v.'s of smaller dimensions.

For root systems $R$ we will follow the definitions and notation in Bourbaki [2]. In particular, $R^{\vee}$ will denote the inverse root system and the Weyl group of $R$ will be denoted by $\mathcal{W}(R)$. We will essentially deal with irreducible and reduced root systems in the sense of [2]; that is, with the root systems denoted by $\mathbf{A}_{\mathbf{n}}, \mathbf{B}_{\mathbf{n}}, \mathbf{C}_{\mathbf{n}}, \mathbf{D}_{\mathbf{n}}, \mathbf{E}_{\mathbf{6}}$, $\mathbf{E}_{7}, \mathbf{E}_{8}, \mathbf{F}_{4}$ and $\mathbf{G}_{2}$.

\section{The General CONSTRUCTION}

In this section we describe a construction of principally polarized abelian varieties admitting a given integral representation of a finite group $G$.

Remark 2.1. Of course, every finite group has integral representations. Indeed, a complex (irreducible) representation of degree $m$ defined over the field $L$ gives rise to a rational (irreducible) representation of degree $m[L: \mathbb{Q}]$, but any (irreducible) rational representation is $\mathbb{Q}$-equivalent to an integral representation.

Every finite group also has faithful integral representations. As an example, we have its regular representation. 
Recall that (see [7]) for any polarized abelian variety $(X=V / \Lambda, H)$, a direct sum decomposition

$$
\Lambda=L_{1} \oplus L_{2}
$$

is called a decomposition for $H$ if $L_{1}$ and $L_{2}$ are isotropic with respect to $\Im H$. Equivalently, if there exists a symplectic basis $\left\{\lambda_{1}, \ldots, \lambda_{n}, \mu_{1}, \ldots, \mu_{n}\right\}$ for $\Lambda$ such that $L_{1}=\left\langle\lambda_{1}, \ldots, \lambda_{n}\right\rangle$ and $L_{2}=\left\langle\mu_{1}, \ldots, \mu_{n}\right\rangle$.

We now define a similar notion for p.p.a.v.'s with $G$-action.

Definition 2.2. Let $G$ denote a finite group and consider a faithful integral representation $\rho$ of $G$. $H$

If $(X=V / \Lambda, H)$ is a p.p.a.v. with $G$-action, we will say that a decomposition for

$$
\Lambda=L_{1} \oplus L_{2}
$$

is a $\rho$-decomposition if both $L_{1}$ and $L_{2}$ are $G$-invariant and $L_{1}$ is $\mathbb{Z} G$-equivalent to $\rho$.

In this case we will say that $(X, H)$ is $\rho$-decomposable.

Remark 2.3. If $(X=V / \Lambda, H)$ is $\rho$-decomposable, then there exists a symplectic canonical basis for $\Lambda$ with respect to which the $G$-action is represented by the symplectic matrices

$$
\left(\begin{array}{cc}
\rho(g) & 0 \\
0 & \rho^{-t}(g)
\end{array}\right), g \in G
$$

where $\rho^{-t}$ is the contragredient representation of $\rho$. The converse is also clearly true.

Also, the $G$-action on the tangent space to $X$ at the origin is (equivalent to) $\rho$.

We will now give a construction for $\rho$-decomposable p.p.a.v.'s.

If $L$ is a $\mathbb{Z} G$-module corresponding to an integral representation $\rho$, we let $U$ denote the real linear span of $L$. For a $\rho(G)$-invariant real inner product $B$ on $U$ that is integral on $L$, we will denote by $L^{*}$ the dual of $L$ with respect to $B$.

Proposition 2.4. Let $G$ denote a finite group and consider a faithful integral representation $\rho$ of $G$ over a $\mathbb{Z} G$ module $L$.

Denote by $U$ (respectively $V$ ) the real (respectively complex) linear span of $L$.

Then, for any $\rho(G)$-invariant real inner product $B$ on $U$ that is integral on $L$, and for any complex number $\tau$ with positive imaginary part, the p.p.a.v. given by

$$
X=X(B, \tau)=V /\left(L+\tau L^{*}\right), \Im H\left(u+\tau u^{\prime}, v+\tau v^{\prime}\right)=B\left(u, v^{\prime}\right)-B\left(v, u^{\prime}\right)
$$

is $\rho$-decomposable.

Furthermore, each such $X$ is isomorphic (as a torus) to a product of elliptic curves.

Proof. Only the last assertion needs to be proven: since $L \subseteq L^{*}$ are integral lattices of the same dimension $n$, then (see [3, p. 221]) there exist a basis $\left\{\ell_{1}, \ldots, \ell_{n}\right\}$ of $L$ 
and positive integers $\left\{d_{j}\right\}_{j=1}^{n}$ such that $d_{j+1}$ divides $d_{j}$ for each $j \in\{1, \ldots, n-1\}$ and such that $\left\{\frac{1}{d_{j}} \ell_{j}\right\}_{j=1}^{n}$ is a basis for $L^{*}$.

It is then clear that the torus $X=V /\left(L+\tau L^{*}\right)$ is isomorphic to the product

$$
E_{\frac{\tau}{d_{1}}} \times \ldots \times E_{\frac{\tau}{d_{n}}}
$$

where $E_{\mu}$ denotes the elliptic curve with modulus $\mu$.

When $\rho$ is an absolutely irreducible representation, we can also prove the converse; that is, we now give a characterization of $\rho$-decomposable p.p.a.v.'s whenever $\rho$ is absolutely irreducible.

Theorem 2.5. If in Proposition 2.4 the representation $\rho$ is absolutely irreducible, then every $\rho$-decomposable p.p.a.v. is of the form described there.

Proof. Let $B$ denote a fixed real inner product on the real span of $L$, with $B$ integral on $L$. For any $\tau$ in $\mathbb{H}_{1}$ we can apply the construction of Proposition 2.4 to find a $\rho$-decomposable p.p.a.v. corresponding to $X(B, \tau)$.

Since $\rho$ is absolutely irreducible, any $\rho(G)$-invariant inner product on the real linear span of $L_{1}$ is a positive constant multiple of $B$ [2, p. 66]; therefore, any positive multiple $a B$ (integral on $L$ ) together with any $\tau$ in $\mathbb{H}_{1}$ give rise (via the construction described in Proposition 2.4) to the $\rho$-decomposable p.p.a.v. corresponding to $X(a B, \tau)$, which is naturally isomorphic to $X\left(B, \frac{1}{a} \tau\right)$.

Given a $\rho$-decomposable p.p.a.v. $(X=V / \Lambda, H)$, without loss of generality we assume that there exists a symplectic basis $\left\{\lambda_{1}, \ldots, \lambda_{n}, \mu_{1}, \ldots, \mu_{n}\right\}$ for $\Lambda$ such that $L_{2}=\left\langle\mu_{1}, \ldots, \mu_{n}\right\rangle$ and

$$
L=L_{1}=\left\langle\lambda_{1}, \ldots, \lambda_{n}\right\rangle .
$$

Then the Riemann matrix $Z=W+i Y$ for $X$ with respect to this basis is fixed under the symplectic action (2.1), and therefore $Y^{-1}$, the inverse of $Y$, yields a $\rho(G)$-invariant inner product on the real linear span of $L$, which is then a positive constant multiple of $B$.

Furthermore, $W Y^{-1}$ is a real endomorphism of the real linear span of $L$ commuting with $\rho$, and therefore a multiple of the identity.

We have thus proven that $Z$ is a complex multiple of the inverse of the matrix of $B$ with respect to the basis $\lambda$, where the scalar lies in $\mathbb{H}_{1}$.

The next result follows immediately from the proof of the theorem.

Corollary 2.6. Let $G$ denote a finite group and consider a faithful integral representation absolutely irreducible $\rho$ of $G$ on a $\mathbb{Z} G$-module $L$.

Then the family of $\rho$-decomposable p.p.a.v.'s is parameterized by the family of Riemann matrices

$$
Z_{\tau}=\tau Z_{0}, \tau \in \mathbb{H}_{1},
$$

where $Z_{0}$ is the inverse matrix of a real inner product on the real span of $L$ which is integral on $L$, with respect to an adequate basis for $L$. 
Remark 2.7. Note that the Riemann matrices described in (2.2) correspond to the fixed points in Siegel space under the action given in (2.1).

Combining Proposition 2.4 with Theorem 2.5 we obtain the following result.

Corollary 2.8. Each $\rho$-decomposable p.p.a.v. for an absolutely irreducible representation $\rho$ of $G$ is isomorphic (as a torus) to a product of elliptic curves.

Example 2.9. The following is an example of an integral representation whose associated family of p.p.a.v.'s is not one-dimensional, and where there are members of the family that are not invariant under the natural action of any Weyl group in this dimension.

Consider the rational irreducible non-trivial representation of $G=\mathbb{Z} / 5 \mathbb{Z}=\left\langle a: a^{5}\right\rangle$ given by

$$
\rho(a)=\left(\begin{array}{llll}
0 & 0 & 0 & -1 \\
1 & 0 & 0 & -1 \\
0 & 1 & 0 & -1 \\
0 & 0 & 1 & -1
\end{array}\right)
$$

Then the corresponding Riemann matrices are given by

$$
Z=\tau_{1}\left(\begin{array}{cccc}
4 & 3 & 2 & 1 \\
3 & 6 & 4 & 2 \\
2 & 4 & 6 & 3 \\
1 & 2 & 3 & 4
\end{array}\right)+\tau_{2}\left(\begin{array}{cccc}
2 & 2 & 1 & 0 \\
2 & 4 & 3 & 1 \\
1 & 3 & 4 & 2 \\
0 & 1 & 2 & 2
\end{array}\right)
$$

where $\tau_{1}, \tau_{2}$ are complex numbers such that the imaginary part of $Z$ is positive definite.

Note that this representation is the restriction to the subgroup $G$ of the natural representation of the Weyl group corresponding to the root system $\mathbf{A}_{\mathbf{4}}$, and that this is the only Weyl group for an irreducible system of dimension 4 having a subgroup isomorphic to $G$. As we will see later on, the family of p.p.a.v.'s associated to the natural representation of the Weyl group just mentioned is the subfamily of (2.3) obtained by setting $\tau_{2}=0$.

Example 2.10. We give an example of an absolutely irreducible integral representation acting on a one parameter family of p.p.a.v.'s of dimension six (which contains a Jacobian, that of Wiman's curve, see [5] ), with the given action on the tangent space at the origin of each member of the family, and such that there is an invariant sublattice of rank six with no invariant complementary sublattice; that is, these p.p.a.v.'s are not $\rho$-decomposable. 
The p.p.a.v.'s are defined by their Riemann matrices

$$
Z_{\tau}=\left[\begin{array}{cccccc}
3 \tau & -\tau & \tau & -\tau & \tau & -\frac{1}{2} \\
-\tau & 3 \tau & -\tau & -\tau & \frac{1}{2} & \tau \\
\tau & -\tau & 3 \tau & -\frac{1}{2} & -\tau & \tau \\
-\tau & -\tau & -\frac{1}{2} & 3 \tau & -\tau & -\tau \\
\tau & \frac{1}{2} & -\tau & -\tau & 3 \tau & -\tau \\
-\frac{1}{2} & \tau & \tau & -\tau & -\tau & 3 \tau
\end{array}\right]
$$

for $\tau \in \mathbb{H}_{1}$.

The group is $G=S_{5}$, the symmetric group on five letters, and the corresponding integral representation $\rho$ is given as follows. Note that $\rho$ is the unique (complex) irreducible representation of degree six of $S_{5}$.

$$
\begin{gathered}
\rho(1,2,3,4,5)=\left[\begin{array}{rrrrrr}
1 & 1 & 0 & 1 & 0 & 0 \\
-1 & 0 & 1 & 0 & 1 & 0 \\
1 & 0 & 0 & 0 & 0 & 0 \\
0 & -1 & -1 & 0 & 0 & 1 \\
0 & 1 & 0 & 0 & 0 & 0 \\
0 & 0 & 1 & 0 & 0 & 0
\end{array}\right] \\
\rho(1,2)=\left[\begin{array}{rrrrrr}
1 & 0 & 0 & 0 & 0 & 0 \\
0 & 1 & 0 & 0 & 0 & 0 \\
0 & 0 & 1 & 0 & 0 & 0 \\
-1 & -1 & 0 & -1 & 0 & 0 \\
1 & 0 & -1 & 0 & -1 & 0 \\
0 & 1 & 1 & 0 & 0 & -1
\end{array}\right]
\end{gathered}
$$

The associated symplectic action is given by

where

$$
\begin{gathered}
\widetilde{\rho}(1,2,3,4,5)=\left[\begin{array}{cc}
\rho(1,2,3,4,5) & 0 \\
0 & \rho^{-t}(1,2,3,4,5)
\end{array}\right] \\
\widetilde{\rho}(1,2)=\left[\begin{array}{cc}
\rho(1,2) & L(1,2) \\
0 & \rho^{-t}(1,2)
\end{array}\right]
\end{gathered}
$$

$$
L(1,2)=\left[\begin{array}{rrrrrr}
0 & 0 & 0 & 0 & 0 & 1 \\
0 & 0 & 0 & 0 & -1 & 0 \\
0 & 0 & 0 & 1 & 0 & 0 \\
0 & 0 & -1 & 0 & 1 & -1 \\
0 & 1 & 0 & -1 & 0 & 1 \\
-1 & 0 & 0 & 1 & -1 & 0
\end{array}\right]
$$


The Riemann matrices $Z_{\tau}$ are the only Riemann matrices invariant under the given action, and a calculation shows that the given symplectic representation is not even integrally equivalent to any integral representation of the following form

$$
\left(\begin{array}{cc}
U(g) & 0 \\
0 & U(g)^{-t}
\end{array}\right) .
$$

\section{The construction for Weyl groups}

For each Weyl group $\mathcal{W}$ there is a natural integral faithful representation $\rho$, generated by the reflections on the roots. Furthermore, this representation is absolutely irreducible if the associated root system is irreducible and reduced.

Therefore, in this case we may apply Theorem 2.5] to find the family of $\rho$-decomposable p.p.a.v.'s, which we also know is parameterized by $\mathbb{H}_{1}$.

We summarize this information in the next proposition, where we include the Riemann matrices for each family for the sake of completeness.

Proposition 3.1. Let $R$ be an irreducible, reduced root system of dimension $n$, and let $\mathcal{W}(R)$ be its Weyl group.

$W e$ denote the corresponding canonical basis for the root system $(V, R)$ by $\mathcal{B}$, and consider the integral representation $\rho$ of dimension $n$ of $\mathcal{W}(R)$ generated by the reflections associated to the elements of $\mathcal{B}$

Then the family of $\rho$-decomposable p.p.a.v.'s is one-dimensional, and parameterized by the family of Riemann matrices $\mathbb{H}(R)$ in $\mathbb{H}_{n}$ associated to $\rho$ by Corollary 2.6 .

The corresponding Riemann matrices are given by

$$
\mathbb{H}(R)=\left\{Z_{\tau}=\tau Z_{0}: \tau \in \mathbb{H}_{1}\right\}
$$

where $Z_{0}$ is given in Table 1 .

The proof of Proposition 3.1 follows Table 1. Note that since $Z_{0}$ is symmetric, we give only the upper half of the matrix. 
Table 1: The Riemann matrices

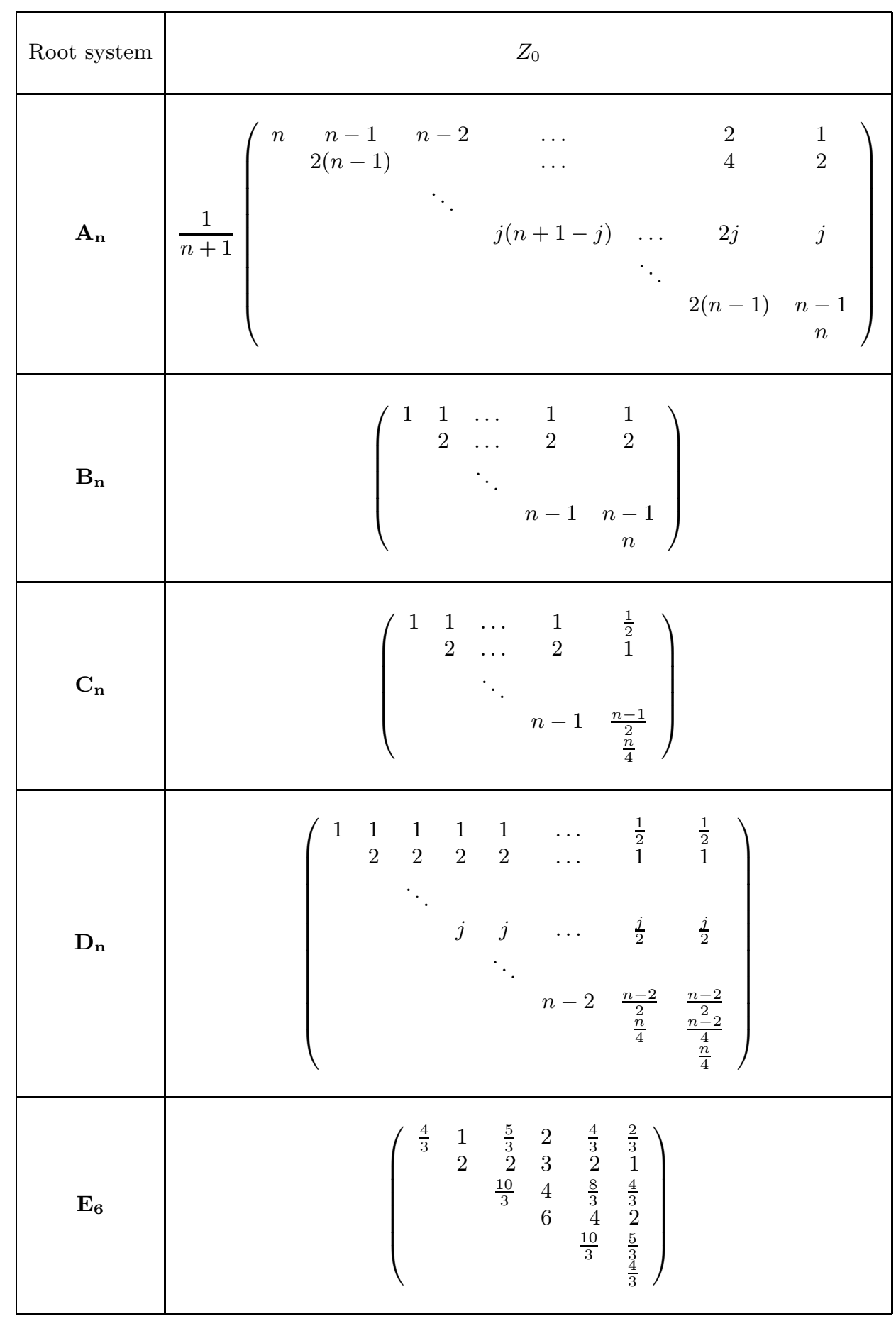


Table 1: (continued)

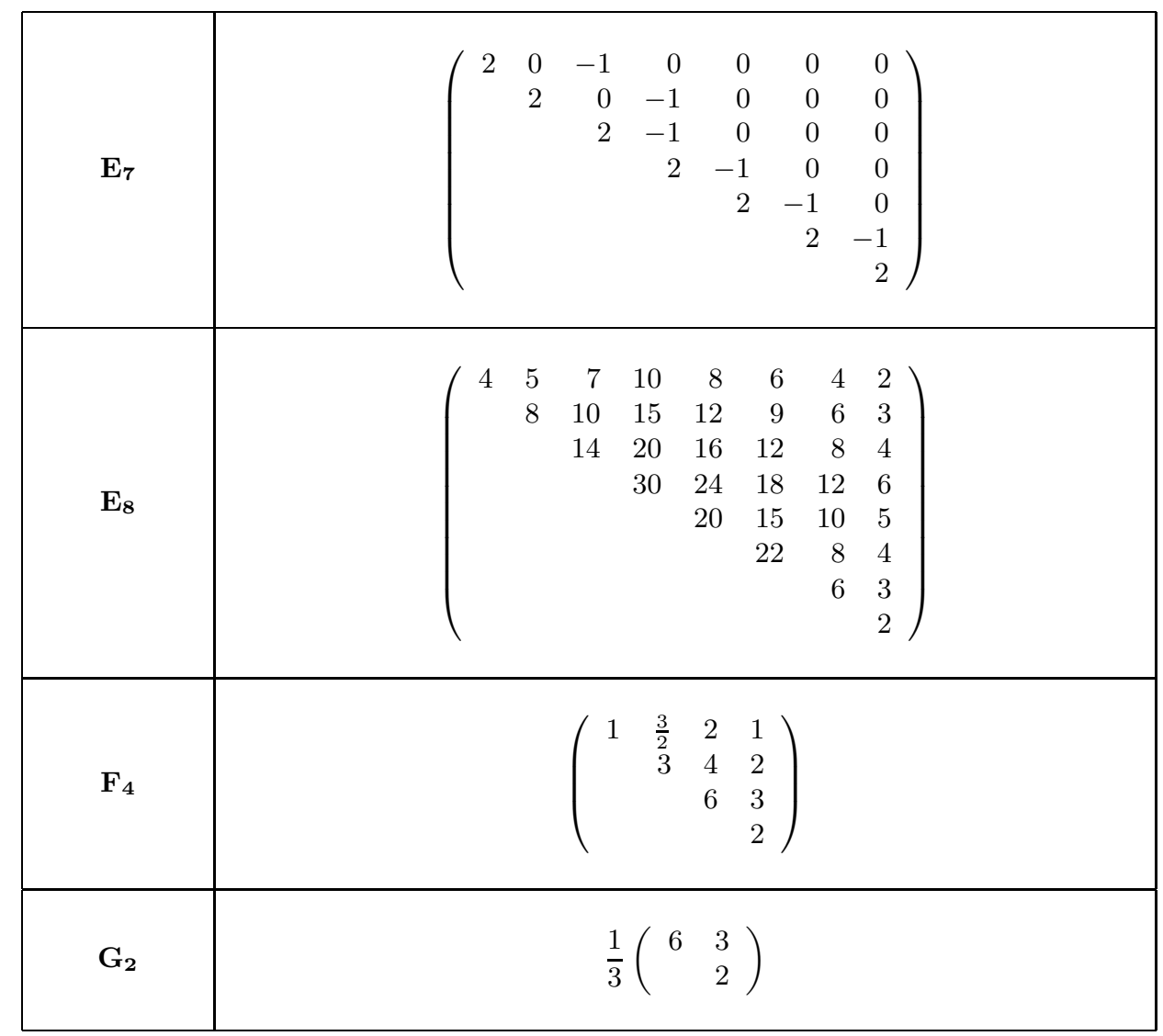

Proof of Proposition [3.1. We follow the notation of [2]; especially see Planches I through IX. All matrices will be given with respect to the basis $\mathcal{B}=\left\{\alpha_{1}, \ldots, \alpha_{n}\right\}$.

Let $C$ denote the Cartan matrix of $R$ given by

$$
C=\left(c_{i j}\right)=\left(2 \frac{\left(\alpha_{i}, \alpha_{j}\right)}{\left(\alpha_{j}, \alpha_{j}\right)}\right),
$$

and let $D$ denote the $n \times n$ diagonal matrix given by $d_{i i}=\frac{\left(\alpha_{i}, \alpha_{i}\right)}{2}$.

Then an integral matrix $S$ for the $\mathcal{W}(R)$-invariant inner product is given by $S=$ $C D$, which allows us to compute the values for $Z_{0}=S^{-1}$ given in the table.

Remark 3.2. Let $R$ be an irreducible, reduced root system of dimension $n$. It is known ([2]) that the group of automorphisms of $R$, denoted by $\operatorname{Aut}(R)$, is a semidirect product of the corresponding Weyl group $\mathcal{W}(R)$ and a finite group $\Gamma$, where $\Gamma$ is the trivial group except in the following cases. 


$$
\begin{aligned}
& \mathbf{R}=\mathbf{A}_{\mathbf{n}}: \Gamma=\mathbb{Z} / 2 \mathbb{Z} \\
& \mathbf{R}=\mathbf{D}_{4}: \Gamma=S_{3} \\
& \mathbf{R}=\mathbf{D}_{\mathbf{n}}, \mathbf{n}>\mathbf{4}: \Gamma=\mathbb{Z} / 2 \mathbb{Z} \\
& \mathbf{R}=\mathbf{E}_{\mathbf{6}}: \Gamma=\mathbb{Z} / 2 \mathbb{Z}
\end{aligned}
$$

A case by case computation allows us to prove the following result.

Corollary 3.3. Let $R$ be an irreducible, reduced root system of dimension $n$.

Denote by $\operatorname{Aut}(R)$ the group of automorphisms of $R$ and by $\widetilde{\rho}$ the natural integral representation of $\operatorname{Aut}(R)$ extending the representation $\rho$ of the Weyl group $\mathcal{W}(R)$.

Then the family of $\widetilde{\rho}$-decomposable p.p.a.v.'s coincides with the family of $\rho$-decomposable p.p.a.v.'s.

More explicitly, each $Z_{\tau} \in \mathbb{H}(R)$ is fixed under the symplectic action (2.1) induced by $\widetilde{\rho}$.

Definition 3.4. Let $R$ be an irreducible, reduced root system of dimension $n$, and let $\mathcal{W}(R)$ be its Weyl group.

Consider the family $\mathbb{H}(R)$ of Riemann matrices in $\mathbb{H}_{n}$ associated to $\rho(\mathcal{W}(R))$ by Proposition 2.4.

For each $Z_{\tau}$ in $\mathbb{H}(R)$ we will denote by $A_{\tau}=\left(X_{\tau}, H_{\tau}\right)$ the corresponding $\rho$-decomposable p.p.a.v., and by $\mathcal{A}(R)$ the corresponding family in $\mathcal{A}_{n}$.

Note that each $A_{\tau}$ is $\widetilde{\rho}$-decomposable, for $\widetilde{\rho}$ as in Corollary 3.3 .

Proposition 3.5. With the notation of Proposition [3.1, the following results hold for each $\tau$ in $\mathbb{H}_{1}$, where the corresponding isomorphisms are as p.p.a.v.'s.

(1) $A_{\tau} \in \mathbb{H}\left(\mathbf{D}_{\mathbf{n}}\right)$ is isomorphic to $A_{\tau} \in \mathbb{H}\left(\mathbf{C}_{\mathbf{n}}\right)$ for each $n$,

(2) $A_{\tau} \in \mathbb{H}\left(\mathbf{F}_{\mathbf{4}}\right)$ is isomorphic to $A_{\tau} \in \mathbb{H}\left(\mathbf{D}_{\mathbf{4}}\right)$, and

(3) $A_{\tau} \in \mathbb{H}\left(\mathbf{G}_{\mathbf{2}}\right)$ is isomorphic to $A_{\tau} \in \mathbb{H}\left(\mathbf{A}_{\mathbf{2}}\right)$.

Proof. It is easy to verify that, in each of the above cases, the integral representations $\widetilde{\rho}$ considered in Corollary 3.3 are isomorphic over $\mathbb{Z}$ for the corresponding groups $\operatorname{Aut}(R)$.

Remark 3.6. Alternatively, the results of Proposition 3.5 may be proved by explicit computations: to show each member of a family $F_{1}=\mathbb{H}\left(\mathbf{R}_{\mathbf{1}}\right)$ is isomorphic to the corresponding element of the family $F_{2}=\mathbb{H}\left(\mathbf{R}_{\mathbf{2}}\right)$, we find an invertible complex $n \times n$ matrix $A$ and a symplectic $2 n \times 2 n$ matrix $M$ such that the following equation holds for each $Z_{\tau}=\tau Z_{1}$ in $F_{1}$ and each $\widetilde{Z_{\tau}}=\tau Z_{2}$ in $F_{2}$.

$$
A\left(\begin{array}{ll}
I_{n} & Z_{\tau}
\end{array}\right)=\left(\begin{array}{ll}
I_{n} & \widetilde{Z_{\tau}}
\end{array}\right) M
$$


In fact, in each case we can choose $A$ to be unimodular (integral) and $M$ to be of

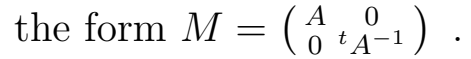

For $F_{1}=\mathbb{H}\left(\mathbf{D}_{\mathbf{n}}\right)$ and $F_{2}=\mathbb{H}\left(\mathbf{C}_{\mathbf{n}}\right)$ we let $A=\left(\begin{array}{rrrr}I_{n-2} & & 0 \\ 0 & 1 & 1 & -1 \\ 0 & 0 & 1 & 0\end{array}\right)$.

For $F_{1}=\mathbb{H}\left(\mathbf{D}_{4}\right)$ and $F_{2}=\mathbb{H}\left(\mathbf{F}_{4}\right)$ we let $A=\left(\begin{array}{cccc}0 & 0 & 0 & 1 \\ 1 & 0 & 0 & 1 \\ 1 & 0 & 1 & 1 \\ 0 & 1 & 0 & 0\end{array}\right)$.

For $F_{1}=\mathbb{H}\left(\mathbf{G}_{\mathbf{2}}\right)$ and $F_{2}=\mathbb{H}\left(\mathbf{A}_{\mathbf{2}}\right)$ we let $A=\left(\begin{array}{rr}1 & -2 \\ 0 & 1\end{array}\right)$.

Remark 3.7. It will be a consequence of Proposition 4.1, Corollary 4.3 and Theorem 4.5 that there no other isomorphisms between different families of type $\mathbb{H}(\mathbf{R})$.

Remark 3.8. The family $\mathbb{H}\left(\mathbf{A}_{\mathbf{n}}\right)$ was discussed in a different context in [6]. The corresponding Riemann matrices mentioned there are of the following form

$$
\widetilde{Z}_{\tau}=\tau\left(\begin{array}{rrrr}
n & -1 & \ldots & -1 \\
-1 & n & & \vdots \\
& & \ddots & -1 \\
-1 & \cdots & -1 & n
\end{array}\right)
$$

This family is of course isomorphic to our family; as above, it is enough to consider the unimodular $n \times n$ matrix

$$
A=\left(\begin{array}{rrrrr}
1 & 0 & 0 & 0 & \cdots \\
-1 & 1 & 0 & 0 & \ldots \\
0 & -1 & 1 & 0 & \cdots \\
\vdots & & \ddots & \ddots & \\
0 & \ldots & & -1 & 1
\end{array}\right)
$$

and verify that Equation (3.1) is satisfied with $M=\left(\begin{array}{cc}A & 0 \\ 0 & A^{-t}\end{array}\right)$.

Remark 3.9. If $R$ is an irreducible, reduced root system we can also perform the construction in Theorem 2.5 for the natural integral representation associated to the lattice generated by $R^{\vee}$, the inverse root system.

A related construction is the following. Let $E$ be a generic elliptic curve and $R$ an irreducible, reduced root system of rank $n$, with $Q$ the lattice generated by $R$ or $R^{\vee}$. Then $X \simeq Q \otimes E$ is an $n$-dimensional complex torus isomorphic (as a complex torus) to the $n$-fold product of the elliptic curve $E$ and the Weyl group $\mathcal{W}(R)$ acts naturally on $X$. The polarization $P$ on $E$ induces an equivariant polarization $P_{X}$ on $X$ which is, in general, not principal. 
Such abelian varieties have been discussed earlier in 8 for the case $Q^{\vee}$ the lattice generated by $R^{\vee}$. One can verify that the natural equivariant polarization induced on $X \simeq Q^{\vee} \otimes E$ is of respective degree $n+1,4,1,4,3,2,1,4$ and 3, corresponding to the system $\mathbf{A}_{\mathbf{n}}, \mathbf{B}_{\mathbf{n}}, \mathbf{C}_{\mathbf{n}}, \mathbf{D}_{\mathbf{n}}, \mathbf{E}_{\mathbf{6}}, \mathbf{E}_{\mathbf{8}}, \mathbf{F}_{\mathbf{4}}$ and $\mathbf{G}_{\mathbf{2}}$.

In Section 5 we will make explicit the relation between these polarized abelian varieties and our corresponding principally polarized abelian varieties.

\section{IrREDUCibility of the ABELIAN VARIETIES ASSOCIATED TO WEYL GROUPS}

In this section we answer the question of irreducibility of the p.p.a.v.'s admitting the natural action of the Weyl groups constructed in Proposition 3.1.

We already know, from Corollary 2.8, that when considered as complex tori they are all isomorphic to the product of elliptic curves. Our next result gives the explicit decomposition in each case.

Proposition 4.1. Let $R$ be an irreducible, reduced root system and consider the corresponding family of p.p.a.v.'s $\mathcal{A}(R)$ as per Definition 3.4.

Then each $A_{\tau}$ in $\mathcal{A}(R)$ is isomorphic (as a complex torus) to a product of elliptic curves, as follows. 
Table 2: Decomposition as complex tori

\begin{tabular}{|c|c|c|}
\hline Root system & Decomposition for $A_{\tau}$ & Case \\
\hline $\mathbf{A}_{\mathbf{n}}$ & $E_{\tau}^{n-1} \times E_{\tau /(n+1)}$ & any $n$ \\
\hline $\mathbf{B}_{\mathbf{n}}$ & $E_{\tau}^{n}$ & any $n$ \\
\hline $\mathbf{C}_{\mathbf{n}}, \mathbf{D}_{\mathbf{n}}$ & $E_{\tau}^{n-1} \times E_{\tau / 4}$ & n odd \\
& $E_{\tau}^{n-2} \times E_{\tau / 2}^{2}$ & $n$ even \\
\hline $\mathbf{E}_{\mathbf{6}}$ & $E_{\tau}^{5} \times E_{\tau / 3}$ & \\
\hline $\mathbf{E}_{\mathbf{7}}$ & $E_{\tau}^{6} \times E_{\tau / 2}$ & \\
\hline $\mathbf{E}_{\mathbf{8}}$ & $E_{\tau}^{8}$ & \\
\hline $\mathbf{F}_{\mathbf{4}}$ & $E_{\tau}^{2} \times E_{\tau / 2}^{2}$ & \\
\hline $\mathbf{G}_{\mathbf{2}}$ & & \\
\hline
\end{tabular}

Proof. A case by case calculation of the integers $d_{j}$ appearing in the proof of Proposition 2.4 gives the result.

Remark 4.2. Alternatively, given a Riemann matrix $Z_{\tau}$ in $\mathbb{H}(R)$, we can find explicit $n \times n$ unimodular matrices $F$ and $M$ and a diagonal $n \times n$ matrix $d$ (with positive entries in the diagonal) which satisfy the following equation

$$
F\left(\begin{array}{ll}
I_{n} & Z_{\tau}
\end{array}\right)=\left(\begin{array}{ll}
I_{n} & \tau d
\end{array}\right)\left(\begin{array}{cc}
F & 0 \\
0 & M
\end{array}\right)
$$

We illustrate with the following example, which will give extra information. 
Let $R=\mathbf{B}_{\mathbf{n}}$. In this case, let

$$
F=\left(\begin{array}{rrrrr}
1 & 0 & \ldots & 0 & 0 \\
-1 & 1 & \ldots & 0 & 0 \\
& & \ddots & & \\
0 & & \ldots & 1 & 0 \\
0 & \ldots & -1 & 1
\end{array}\right), d=I_{n}, \quad \text { and } M=F S^{-1}
$$

and note that equation (4.1) is satisfied.

This calculation provides the first result about the irreducibility of these p.p.a.v.'s, as follows.

Corollary 4.3. For every natural number $n$ and every $\tau$ in $\mathbb{H}_{1}$, the p.p.a.v. $A_{\tau}$ in $\mathcal{A}\left(\mathbf{B}_{\mathbf{n}}\right)$ is isomorphic as a p.p.a.v. to the product of $n$-times the elliptic curve $E_{\tau}$.

Proof. Observe that $M=F^{-t}$ for the explicit matrices given above.

Therefore the matrix $\left(\begin{array}{cc}F & 0 \\ 0 & M\end{array}\right)$ appearing in the equivalence of Remark 4.2 is symplectic, which finishes the proof.

About the case $R=\mathbf{A}_{\mathbf{n}}$, the following result may be found in [6] (see also Remark 3.8).

Proposition 4.4. Consider the root system of type $\mathbf{A}_{n}$ and denote by $\mathcal{A}\left(\mathbf{A}_{\mathbf{n}}\right)$ the corresponding family of p.p.a.v.'s of dimension $n$ given in Definition 3.4.

Then each $A_{\tau}$ in $\mathcal{A}\left(\mathbf{A}_{\mathbf{n}}\right)$ is irreducible as a p.p.a.v., except when $n=2$ and $\tau$ is equivalent to $\tau_{0}=\frac{-3+i \sqrt{3}}{6} \bmod \Gamma_{0}(3)$, in which case $A_{\tau}$ is isomorphic as a p.p.a.v. to $E_{\omega} \times E_{\omega}$, where $E_{\omega}$ is the elliptic curve with $j(\omega)=0$.

We will now answer the question of irreducibility as p.p.a.v.'s for the other irreducible root systems, where by the isomorphisms given in Proposition 3.5 we only need to consider the cases $R=\mathbf{C}_{\mathbf{n}}, \mathbf{E}_{\mathbf{6}}, \mathbf{E}_{\mathbf{7}}$ and $\mathbf{E}_{\mathbf{8}}$. Note that the proof applies to the case $\mathbf{A}_{n}$ as well.

Theorem 4.5. For each of the root systems $R=\mathbf{C}_{\mathbf{n}}(n \geq 3), \mathbf{E}_{\mathbf{6}}, \mathbf{E}_{\mathbf{7}}$ and $\mathbf{E}_{\mathbf{8}}$ consider the respective family of p.p.a.v.'s $\mathcal{A}(R)$ given in Definition 3.4.

Then each $\left(X_{\tau}, H_{\tau}\right)$ in $\mathcal{A}(R)$ is irreducible as a p.p.a.v., except for some exceptional values of $\tau$ in the case $R=\mathbf{C}_{\mathbf{n}}$.

Proof. Suppose that $\left(X_{\tau}, H_{\tau}\right)=(X, H)$ is isomorphic as a p.p.a.v. to a product of p.p.a.v.'s as follows

$$
(X, H) \simeq\left(X_{1}, H_{1}\right) \times\left(X_{2}, H_{2}\right) \times \ldots \times\left(X_{k}, H_{k}\right),
$$

with $k>1$. 
Then the tangent space at the origin for $X, T(X)$, decomposes accordingly

$$
T(X) \simeq \oplus_{j=1}^{k} T\left(X_{j}\right)
$$

Furthermore, the corresponding Weyl group preserves this decomposition, and therefore the group is imprimitive.

But then the root system under consideration must be $\mathbf{C}_{\mathbf{n}}$ (see [4]); furthermore all $\left(X_{j}, H_{j}\right)$ are isomorphic to each other, and $\operatorname{dim} X_{j}=1$.

That is, $X \simeq X_{1}^{n}$, where $X_{1}$ is an elliptic curve. But by Proposition 4.1 we also have that

$$
X \simeq E_{\tau}^{n-1} \times E_{\tau / 4} \text { for } n \text { odd }
$$

and that

$$
X \simeq E_{\tau}^{n-2} \times E_{\tau / 2} \times E_{\tau / 2} \text { for } n \text { even }
$$

as complex tori.

This finishes the proof, since then the only values of $\tau$ for which $\left(X_{\tau}, H_{\tau}\right)$ may be reducible are for the case of the root system $\mathbf{C}_{\mathbf{n}}$ and $E_{\tau} \simeq E_{\tau / 4}$, for $n$ odd, and $E_{\tau} \simeq E_{\tau / 2}$, for $n$ even.

Remark 4.6. The Jacobian variety $J(C)$ of a complex curve $C$ of genus $n$ is an irreducible principally polarized abelian variety of dimension $n$ (irreducibility of the theta divisor). Nevertheless, it is an interesting problem to find Jacobians isomorphic as tori (and not only isogenous) to products of elliptic curves.

As all the p.p.a.v.'s in the families associated to Weyl groups are isomorphic to products of elliptic curves, a necessary condition for a family $\mathcal{A}(R)$ in the moduli space $\mathcal{A}_{n}$ of p.p.a.v.'s of dimension $n$ to intersect the Jacobian locus $\mathcal{J}_{n}$ is given by the Hurwitz and Torelli theorems.

For example, these condition are satisfied for the root systems $\mathbf{A}_{\mathbf{4}}$ and $\mathbf{A}_{\mathbf{5}}$, and in those cases the corresponding intersection is characterized in [1], [6] and [10].

\section{The ABELIAN VARIETIES FAMILIES FOR WEYL GROUPS ARE MODULAR CURVES}

In this section we will show that the families $\mathcal{A}(R)$ constructed in Section 3 may be described as modular curves in the corresponding moduli space of principally polarized abelian varieties.

Remark 5.1. Let $R$ be an irreducible root system and denote by $G$ the natural symplectic representation of either its Weyl group $\mathcal{W}(R)$ given by (2.1).

Denote by $C(G)$ and $N(G)$ the respective centralizer and normalizer of $G$ inside the corresponding symplectic group, and by $\operatorname{Aut}(G)$ the group of automorphisms of $G$ that may be realized inside the symplectic group.

Then we have the following exact sequence of groups

$$
1 \rightarrow C(G) \stackrel{i}{\rightarrow} N(G) \stackrel{\varphi}{\rightarrow} \operatorname{Aut}(G) \rightarrow 1
$$


where $i$ denotes the inclusion and $\varphi(M)$ is conjugation by $M$ for each $M$ in $N(G)$. Furthermore, the family $\mathcal{A}(R)$ in $\mathcal{A}_{n}$ is given by $\mathbb{H}(R) / C(G)$, so we need to describe $C(G)$, which we do next.

Proposition 5.2. Let $R$ be an irreducible root system of dimension $n$ and consider the symplectic representation of its Weyl group $\mathcal{W}(R)$ associated to the natural basis for the roots, as in (2.1).

Then its centralizer in $\operatorname{Sp}(2 n, \mathbb{Z})$ is isomorphic to a subgroup of $\operatorname{PSL}(2, \mathbb{Z})=\Gamma$, as follows.

Table 3: Centralizers for $\mathcal{W}(R)$

\begin{tabular}{|c|c|c|}
\hline Root system & Centralizer & Case \\
\hline $\mathbf{A}_{\mathbf{n}}$ & $\Gamma^{0}(n+1)$ & any $n$ \\
\hline $\mathbf{B}_{\mathbf{n}}, \mathbf{E}_{\mathbf{8}}$ & $\Gamma$ & any $n$ \\
\hline $\mathbf{C}_{\mathbf{n}}, \mathbf{D}_{\mathbf{n}}$ & $\Gamma^{0}(4)$ & n odd \\
& $\Gamma^{0}(2)$ & $n$ even \\
\hline $\mathbf{E}_{\mathbf{6}}, \mathbf{G}_{\mathbf{2}}$ & $\Gamma^{0}(3)$ & \\
\hline $\mathbf{E}_{\mathbf{7}}, \mathbf{F}_{\mathbf{4}}$ & $\Gamma^{0}(2)$ & \\
\hline
\end{tabular}

Proof. We observe that $\Gamma=\operatorname{PSL}(2, \mathbb{Z})$ acts naturally on $\mathbb{Z}^{2} \otimes L^{*}=L^{*} \oplus L^{*}$, and that the centralizer we are looking for is isomorphic to the stabilizer of $L \oplus L^{*}$ under this action, which is then computed using Table 2.

Remark 5.3. Alternatively, since the natural integral representation of each Weyl group is absolutely irreducible, it follows that each matrix in the centralizer has the following form

$$
\left(\begin{array}{cc}
a I_{n} & b Z_{0} \\
c Z_{0}^{-1} & d I_{n}
\end{array}\right)
$$


with $a, b, c, d \in \mathbb{Z}$ and $a d-b c=1$, and $Z_{0}$ as in Table 3.1. Noting that $Z_{0}^{-1}$ is an integral matrix, the specific extra conditions on $b$ in each case follow from Table 3.1 .

We can now parameterize each family $\mathcal{A}(R)$ as follows.

Theorem 5.4. Let $R$ be an irreducible root system of dimension $n$.

Then the set $\mathcal{A}(R)$ of isomorphism classes of p.p.a.v.'s in $\mathcal{A}_{n}$ which are $\rho$-decomposable for the natural action of the corresponding Weyl group $\mathcal{W}(R)$, is parameterized by a modular curve.

More precisely, we have the following result.

Table 4: Parameterizing the families $\mathcal{A}(R)$

\begin{tabular}{|c|c|c|}
\hline Root system $R$ & $\mathcal{A}(R)$ & Case \\
\hline $\mathbf{A}_{\mathbf{n}}$ & $\mathbb{H}_{1} / \Gamma^{0}(n+1)$ & any $n$ \\
\hline $\mathbf{B}_{\mathbf{n}}, \mathbf{E}_{\mathbf{8}}$ & $\mathbb{H}_{1} / \Gamma$ & any $n$ \\
\hline $\mathbf{C}_{\mathbf{n}}, \mathbf{D}_{\mathbf{n}}$ & $\mathbb{H}_{1} / \Gamma^{0}(4)$ & n odd \\
& $\mathbb{H}_{1} / \Gamma^{0}(2)$ & n even \\
\hline $\mathbf{E}_{\mathbf{6}}, \mathbf{G}_{\mathbf{2}}$ & $\mathbb{H}_{1} / \Gamma^{0}(3)$ & \\
\hline $\mathbf{E}_{\mathbf{7}}, \mathbf{F}_{\mathbf{4}}$ & $\mathbb{H}_{1} / \Gamma^{0}(2)$ & \\
\hline
\end{tabular}

Proof. Consider an irreducible root system $R$ of dimension $n$ and denote by $\mathbb{H}(R)$ the family of Riemann matrices in $\mathbb{H}_{n}$ constructed in Proposition 3.1 .

It follows that the natural morphism

$$
\mathbb{H}(R) \rightarrow \mathbb{H}_{1}
$$

which sends $Z_{\tau}$ to $\tau$ is equivariant under the action of the centralizer of $\operatorname{Aut}(R)$ in $\operatorname{Sp}(2 n, \mathbb{Z})$. 
Since this centralizer was described in Proposition 5.2 as a subgroup $\Gamma_{1}$ of $\operatorname{PSL}(2, \mathbb{Z})$, there is an induced injective morphism

$$
\mathbb{H}(R) / \text { centralizer } \rightarrow \mathbb{H}_{1} / \Gamma_{1} \text {. }
$$

Conversely, let $(E, U)$ be a point of the modular curve $\mathbb{H}_{1} / \Gamma_{1}$. Then $E$ is an elliptic curve and $U$ is a cyclic subgroup of the appropriate order in $E$.

The construction of a principally polarized abelian variety in our family starting from these data is obtained following the idea discussed in [6] for the case $\mathbf{A}_{\mathbf{n}}$ as follows. Start by constructing an abelian variety $X$ of dimension $n$, naturally associated to the root system and the elliptic curve, and with the natural action of the Weyl group on $X$. There is a natural equivariant polarization $P_{X}$ on $X$ such that we can embed the subgroup $U$ inside the kernel of $P_{X}$, obtaining a cyclic group which is both invariant under the group action and totally isotropic for the Weil form. It follows that there is a principal polarization $Q$ on $X / U$ whose inverse image is $P_{X}$.

Since the construction is equivariant for the group action, the principally polarized abelian variety $(X / U, Q)$ is the required one.

The proof is finished by explicit calculations in a case by case analysis, by giving an embedding of each cyclic group $U$ into the kernel of $P_{X}$ with the required properties.

Remark 5.5. We can now complete the description of the relationship between our varieties and the ones constructed in [8].

With the notation given in Remark [3.9, the abelian varieties $X=Q^{\vee} \otimes E$ of $[8]$ coincide with the abelian varieties $X$ constructed in the proof of Theorem 5.4 (for the respective inverse root system). In other words, our p.p.a.v.'s are obtained as quotients by appropriate cyclic subgroups of the abelian varieties given in [8].

Acknowledgement. The authors would like to thank the referee for making numerous suggestions that improved the presentation and for pointing out misprints.

\section{REFERENCES}

[1] Ch. Birkenhake and H.Lange, Complex Tori, Progress in Mathematics 177 Birkhauser (1999).

[2] N. Bourbaki, Éléments de mathématique. Fasc. XXXIV. Groupes et algèbres de Lie. Chapitre IV: Groupes de Coxeter et systèmes de Tits. Chapitre V: Groupes engendrés par des réflexions. Chapitre VI: systèmes de racines. (French) Actualités Scientifiques et Industrielles, No. 1337 Hermann, Paris 1968288 pp.

[3] J. W. S. Cassels, Rational quadratic forms. London Mathematical Society Monographs 13. Academic Press, London-New York, (1978).

[4] M. Geck and G. Malle, Reflection Groups. A Contribution to the Handbook of Algebra arXiv math.RT/0311012

[5] V. González-Aguilera and R. E. Rodríguez, A pencil in $\widetilde{\mathfrak{M}}_{6}$ with three points at the boundary, Geom. Dedicata 42 (1992), no. 3, 255-265. 
[6] V. González-Aguilera and R. E. Rodríguez, Families of irreducible principally polarized abelian varieties isomorphic to a product of elliptic curves, Proc. Amer. Soc. 128 (2000), 629-635.

[7] H. Lange and Ch. Birkenhake, Complex Abelian Varieties, Grundlehren der Mathematischen Wissenschaften 302. Springer-Verlag, Berlin, (1992).

[8] E. Looijenga, Root Systems and Elliptic Curves, Invent. Math. 38 (1976), no. 1, 17-32.

[9] L. Moret-Bailly, Familles de courbes et des variétés abéliennnes sur $\mathbb{P}_{1}$, Astérisque. 86 (1981), 109-124.

[10] G. Riera and R. E. Rodríguez, The period matrix of Bring's curve, Pacific J. Of Math. 154 (1992), 179-200.

[11] R. E. Rodríguez and V. González-Aguilera, Fermat's quartic curve, Klein's curve and the Tetrahedrom, Contemp. Math. 201 (1997), 43-62.

[12] J. P. Serre, Linear representations of finite groups, GTM 42, Springer-Verlag, New York, 1996.

Facultad de Matemáticas, Pontificia Universidad Católica de Chile, Casilla 306, Correo 22, Santiago, Chile

E-mail address: acarocca@mat.puc.cl

Departamento de Matemáticas, Universidad Técnica Federico Santa María, Casilla 110-V, VALPARAÍSO, CHILE

E-mail address: vgonzale@mat.utfsm.cl

Facultad de Matemáticas, Pontificia Universidad Católica de Chile, Casilla 306, Correo 22, Santiago, Chile

E-mail address: rubi@mat.puc.cl 